\title{
Competencias transmedia en estudiantes de Periodismo: producción y edición de contenidos informativos multimedia
}

\author{
Transmedia literacy competences of Journalism students: \\ production and editing of informative multimedia content
}

\section{Habilidades transmídia em estudantes de Jornalismo: produção e edição de conteúdo informativo multimídia}

\section{Santiago Giraldo-Luque \\ Profesor del Departamento de Periodismo y \\ Ciencias de la Comunicación \\ (Universidad Autónoma de Barcelona) \\ http://orcid.org/0000-0003-0024-7081}

$$
\text { España }
$$

\section{Santiago Tejedor}

Profesor del Departamento de Periodismo y

Ciencias de la Comunicación

(Universidad Autónoma de Barcelona)

https://orcid.org/0000-0002-5539-9800

$$
\text { España }
$$

\author{
Marta Portalés-Oliva \\ Investigadora del Departamento de \\ Periodismo y Ciencias de la Comunicación \\ (Universidad Autónoma de Barcelona) \\ http://orcid.org/0000-0002-9143-3551 \\ España
}

\section{Ricardo Carniel-Bugs}

Profesor del Departamento de Periodismo y

Ciencias de la Comunicación

(Universidad Autónoma de Barcelona)

http://orcid.org/0000-0002-9303-9515

España

Fecha de recepción: 24 de agosto de 2019

Fecha de revisión: 26 de enero de 2020

Fecha de aceptación: 15 de marzo de 2020

Fecha de publicación: 1 de julio de 2020

Para citar este artículo: Giraldo-Luque, S., Tejedor, S., Portalés-Oliva, M. y Carniel-Bugs,

R. (2020). Competencias transmedia en estudiantes de Periodismo: producción y edición de contenidos informativos multimedia, Icono 14, 18 (1), 84-110. doi: 10.7195/ri14. v18i1.1445 


\section{MONOGRÁFICO}

\section{Resumen}

El artículo se aproxima al uso que los jóvenes realizan de las herramientas multimedia cuando deciden comunicar profesionalmente un producto periodístico. El estudio está centrado en las nuevas plataformas digitales de contenido periodístico y examina los resultados de una investigación que indaga las competencias del "alfabetismo transmedia" en los estudiantes de Periodismo de la Universidad Autónoma de Barcelona (UAB) a lo largo de varios cursos. A partir de 28 reportajes multimedia, se aplica un análisis de contenido cuantitativo y cualitativo realizado sobre los trabajos presentados por los alumnos, e introduce las opiniones de tres grupos focales con los autores de los proyectos. El estudio demuestra que el alumnado confiere un protagonismo destacado a los elementos visuales relacionados con sus proyectos de curso. Los trabajos analizados demuestran, sin embargo, que los componentes emotivos de las historias desatienden aspectos del periodismo transmedia, y que entre los estudiantes existe una confusión terminológica sobre qué es un producto transmediático. El artículo identifica la prevalencia de una alfabetización instrumental o técnica, ligada al manejo adecuado de imágenes y elementos multimedia, pero no crítica, tanto en el discurso como en los contenidos creados por los estudiantes.

Palabras clave: Transmedia; Competencias periodísticas; Periodismo transmedia; Contenidos informativos; Enseñanza universitaria; Alfabetización mediática

\section{Abstract}

The paper analyses the use of multimedia tools that young people use in order to communicate a journalistic product. The study focuses on the new digital platforms of journalistic content and examines the results of a research that explores the competences of "transmedia literacy" in Journalism students of the Autonomous University of Barcelona (UAB) over several courses. The text examines 28 multimedia reports presented by students through a quantitative and qualitative content analysis, and introduces the opinions of three focus groups with the authors of the projects. The study shows that the students confer a prominent role to the visual elements inside of their own reports. The analyzed works demonstrate, however, that the emotive components of the stories ignore aspects of transmedia journalism, and that among the students there is a terminological confusion about what a transmedia product is. 
The article identifies the prevalence of instrumental or technical literacy, linked to the inclusion of pictures and audio-visual content in their products, but not critical, both in the discourse and in the contents created by the students.

Key Words: Transmedia; Journalistic competences; Transmedia journalism; Informetive content; University education; Media literacy

\section{Resumo}

0 artigo aborda o uso que os jovens fazem das ferramentas multimídia quando decidem comunicar profissionalmente um produto jornalístico. 0 estudo centra-se nas novas plataformas digitais de conteúdo jornalístico e examina os resultados de uma pesquisa que investiga as competências de "literacia transmídia" em estudantes de Jornalismo da Universidade Autónoma de Barcelona (UAB) ao longo de vários cursos. Com base em 28 reportagens multimídia, uma análise de conteúdo quantitativa e qualitativa é realizada sobre os trabalhos apresentados pelos alunos, e contempla as opiniões de três grupos focais com os autores dos projetos. 0 estudo mostra que os alunos dão um papel proeminente aos elementos visuais relacionados aos seus projetos de curso. Os trabalhos analisados mostram, no entanto, que os componentes emocionais das histórias desconsideram aspectos do jornalismo transmídia, e que entre os estudantes há confusão terminológica sobre o que é um produto transmídia. 0 artigo identifica a prevalência da alfabetização instrumental ou técnica, vinculada ao manejo adequado de imagens e elementos multimídia, mas não crítica, tanto no discurso quanto nos conteúdos criados pelos alunos.

Palavras chave: Transmídia; Habilidades jornalísticas; Jornalismo transmídia; Conteúdo informativo; Educação universitária; Alfabetização midiática

\section{Introducción}

La nueva ecología de los medios inaugura una reflexión conceptual y terminológica que afecta a la sociedad $y$, en particular, a los procesos comunicativos. En diferentes ámbitos se ha conformado un entorno en el que la comunicación asume un papel protagónico (Piscitelli, 2002). Desde el planteamiento holístico (Postman, 1970), nos situamos en un escenario de transformaciones en la estructura, en la tipología de los mensajes y 


\section{MONOGRÁFICO}

en los procesos comunicativos. Se trata de una nueva ecología mediática (Scolari, 2013a, p. 1418) que redefine ambientes y que genera claros efectos en los receptores.

La irrupción de las nuevas tecnologías impulsó un proceso acelerado de instrumentalización que introdujo en la sociedad una ideología técnica (Wolton, 2000). Las narrativas transmedia, que se extienden a través de distintas plataformas y medios, ofrecen interesantes alternativas a unos relatos digitales que necesitaban superar el calificativo de multimedia (Jenkins, 2006, p. 16).

Junto al nuevo panorama comunicativo, el otro gran reto se sitúa en el terreno de la educación. La emergencia y la rápida consolidación de la figura del prosumidor (Toffler, 1980) en el marco de una cultura colaborativa (Jenkins, 2008) define un perfil de usuario con una particular concepción, valoración y utilización de la información, las plataformas y los instrumentos comunicativos. En el escenario, presidido por procesos que potencian los intercambios dialógicos, la convergencia y la inteligencia colectiva (Lévy, 2007), emergen nuevas alfabetizaciones que superan la acepción de alfabetismo mediático. El conjunto de cambios genera desafíos tanto para los comunicadores como para los educadores. Piscitelli, Adaime y Binder (2010), Tramullas (2016) o Pérez Tornero y Celot (2009), entre otros, han advertido de la importancia de reformular los procesos educativos para poder incidir de forma crítica y reflexiva en la formación de la denominada generación "millennial" (McCrindle y Wolfinger, 2010, p. 202).

El artículo reflexiona así sobre la adquisición de competencias de "alfabetismo transmedia" en los universitarios a partir de un estudio centrado en estudiantes de Periodismo de cuatro cursos académicos (de 2013 a 2016) del grado de Periodismo de la Universidad Autónoma de Barcelona (UAB). El estudio desarrolla grupos focales con alumnos y analiza cuantitativa y cualitativamente una muestra de proyectos finales de la asignatura de segundo curso denominada "Escritura periodística en multimedia e interactivos", que introduce a los estudiantes en los fundamentos de las narrativas on-line.

La investigación se estructura alrededor de la siguiente pregunta de investigación: “¿Qué parámetros consideran los estudiantes de Periodismo en la concepción y producción periodística de sus relatos transmedia?". Con relación a ella, se han considerado las siguientes hipótesis de trabajo: 
- H1. El alumnado desatiende los aspectos relativos a la selección, contraste y verificación de los recursos multimedia que utilizan en sus trabajos.

- H2. Los estudiantes atesoran una gran capacidad creativa en la ideación de propuestas narrativas con finalidad informativa que superan el modelo secuencial o lineal propio de la lógica del papel.

- H3. Los contenidos audiovisuales y de las redes sociales poseen un papel protagónico en la concepción de los proyectos informativos transmedia.

\subsection{Millennials y transmedia: El relato, el mundo y la experiencia}

Los contenidos multimedia interactivos significaron una revolución (Castells, 2003) que sentó las bases de la denominada cibercultura (Lévy, 1999, 2007). La combinación en un mismo mensaje de atributos textuales, visuales y sonoros - acompañados de la interactividad- multiplicó las opciones de definir y articular todo tipo de relatos. El calificativo de "multimedia" comenzó a acompañar a una gran variedad de proyectos, productos y servicios. Algunos autores, incluso, aludían a un falso multimedia para referirse a mensajes caracterizados por la sobreabundancia de atributos informativos que provocan una pérdida de la eficacia informativa. Posteriormente, el denominado "periodismo mashup" convirtió al periodista en un ensamblador de itinerarios o de "rutas" de naturaleza hipertextual que conectaban con otros recursos on-line existentes en la Red. Esta tipología de contenidos suponía un importante viraje en el proceso de convergencia de los recursos y retomaba la esencia del lejano palimpsesto que ahora pasaba a ser digital, multimedia, interactivo y colaborativo (Tejedor, 2007, p. 19).

Actualmente, el transmedia, término cuyo origen puede atribuirse a Kinder (1991) y su conceptualización a Jenkins (2003), se ha consolidado como un nuevo modo de relato que incorpora una amalgama de medios y de plataformas. El concepto de mundo transmedial (Klastrup y Tosca, 2004, p. 409) alude a un escenario comunicativo donde se generan escenarios y experiencias más allá de los relatos (Costa, 2013) y que destaca por la gran cantidad de aportes y de creaciones de los fans. El transmedia concede, por una parte, un papel protagónico a los usuarios (como auténticos lectoautores) en los procesos de difusión, enriquecimiento y viralización (Scolari, 2013a) de los contenidos (convertidos en historias). Por otra, se aprecia como un proceso que "activa dinámicas comunicativas" y que determina las "reglas que gobiernan los 


\section{MONOGRÁFICO}

flujos comunicativos en el ecosistema digital" (Carrera, et al, 2014, p. 543). Los estudios identifican un acelerado incremento del uso de las plataformas on-line (AIMC, 2017) así como una inclinación hacia los dispositivos y aplicaciones que fomenten la interacción, la horizontalidad y la lógica colaborativa propia de la Web 2.0.

La participación, la interacción y la colaboración de los internautas (Pratten, 2011) son rasgos intrínsecos de los productos y de los procesos transmedia en el marco de un escenario presidido por la generación "millennial" — "Generación del Milenio" o "Generación Y" - y la creciente "Generación Z", que introducen novedades y retos investigativos en la comunicación y la educación (Francese, 2003).

\section{Marco teórico}

Los mensajes transmedia fomentan la readaptación y la integración constante de los contenidos (Marfil-Carmona, 2013, p. 140). De este modo, el usuario (prosumidor) y el creador de la obra comparten competencias: ambos pueden definir, crear, enriquecer, difundir, etc. El enfoque reticular ha encontrado en el ámbito del entretenimiento su principal campo de cultivo. No obstante, los relatos periodísticos o de otros ámbitos han comenzado a incorporar las potencialidades comunicativas que ofrece el enfoque transmediático. Larrondo Ureta (2016, p. 36) considera que el transmedia periodístico y el de ficción comparten aspectos básicos como la convergencia multimedia y la cultura participativa.

Los trabajos académicos sobre el transmedia se han centrado principalmente en el estudio de los tipos de relatos de los productos transmedia y en el rol de las audiencias o fans (Scolari, 2017; Cascajosa y Molina, 2017). Sin embargo, el concepto de "alfabetismo transmedia" (transmedia literacy) apenas ha sido estudiado en su alcance conceptual y en sus principales dimensiones analíticas y operativas, siendo éste uno de los objetivos del I+D+I Horizon 2020 liderado por la Universidad Pompeu Fabra ${ }^{1}$. La investigación plantea la necesidad de proyectar la denominación tradicional de alfabetismo mediático (media literacy) a nuevos escenarios, donde el nuevo concepto recoja la cultura colaborativa, el aprendizaje informal y las competencias que se adquieren fuera de la escuela, además de tener en cuenta competencias como la dimensión emocional, entre otras (Scolari, 2016, p.19). 
MONOGRÁFICO

\begin{tabular}{|l|c|c|c|}
\hline Alfabetismo & $\begin{array}{c}\text { Alfabetismo } \\
\text { mediático }\end{array}$ & $\begin{array}{c}\text { Alfabetismo } \\
\text { transmedia }\end{array}$ \\
\hline Soporte mediático & $\begin{array}{c}\text { Libros y otros textos } \\
\text { impresos }\end{array}$ & Broadcasting (TV) & Redes digitales \\
\hline Semiótica del medio & Verbal (leer/escribir) & $\begin{array}{c}\text { Multimodal (medios } \\
\text { audiovisuales) }\end{array}$ & $\begin{array}{c}\text { Multimodal (medios } \\
\text { interactivos / } \\
\text { transmedia) }\end{array}$ \\
\hline $\begin{array}{l}\text { Interpelación del } \\
\text { sujeto }\end{array}$ & Como iletrado & $\begin{array}{c}\text { Como consumidor } \\
\text { (espectador) }\end{array}$ & Como prosumidor \\
\hline Objetivo de la acción & Desarrollar & $\begin{array}{c}\text { Desarrollar espec- } \\
\text { tadores críticos y } \\
\text { en menor medida } \\
\text { productores }\end{array}$ & $\begin{array}{c}\text { Desarrollar prosumidores } \\
\text { criticos }\end{array}$ \\
\hline Entorno de aprendizaje & Formal (escuela) & Formal (escuela) & Informal (extraescolar) \\
\hline Referencias teóricas & Lingüística & $\begin{array}{c}\text { Teoría de los } \\
\text { efectos de los } \\
\text { medios }\end{array}$ & Estudios culturales / \\
Ecología de los medios
\end{tabular}

Tabla 1: Alfabetismo, alfabetismo mediático y alfabetismo transmedia.

Fuente: Elaboración propia a partir de Scolari (2016, p. 21).

Existe una tendencia a establecer alfabetizaciones específicas, como la news literacy, film literacy, visual literacy, entre otras, que formarían parte de la alfabetización mediática (UNESC0, 2013, p. 27), la cual actúa como concepto paraguas y recoge el conjunto de competencias que deben presentar los usuarios para desenvolverse con solvencia y criticidad en un universo de medios, géneros y formatos. El transmedia formaría también parte de la alfabetización mediática en el contexto actual (Pérez Tornero y Celot, 2009, p. 14).

Progresivamente, aparecen concepciones y propuestas terminológicas nuevas, como las "alfabetizaciones múltiples", utilizada por el gobierno finlandés en la última reforma educativa (Palsa y Ruokamo, 2017). Las alfabetizaciones múltiples "representan una multiplicidad de canales de comunicación, tanto impresos como electrónicos, donde lo textual es a menudo relacionado con lo visual, el audio, lo espacial [...]. El texto es considerado cada vez más multimodal" (Leino, Linnakylä y Malin, 2004, p. 254). 


\section{MONOGRÁFICO}

Por lo tanto, el vínculo entre las alfabetizaciones múltiples y el alfabetismo transmedia quedaría latente. En esta línea de lo múltiple, Jenkins elaboró las New Media Literacies (2006, pp. 170-171), donde el transmedia era una de ellas y cuyo objetivo era definir las competencias necesarias para que los jóvenes incidieran en la cultura de la participación².

The New Media Literacies constitute the core cultural competencies and social skills that young people need in our new media landscape. We call them "literacies," but they change the focus of literacy from one of individual expression to one of community involvement. They build on the foundation of traditional literacy, research skills, technical skills, and critical analysis skills taught in the classroom (Jenkins, 2006, p. 13).

Por ello, la alfabetización transmedia invita a repensar las competencias necesarias para el siglo XXI a partir de las dinámicas culturales de los jóvenes en contraposición con la educación formal, como afirma Vázquez García (2015, p. 23) la universidad no puede quedar atrás en la consolidación de nuevos esquemas de competencias. Estas dinámicas culturales están relacionadas a las competencias necesarias en entornos profesionales, como la organización colectiva a través de las redes y la gestión "multitasking" de informaciones (Pérez Latorre, 2015). El papel que desempeña el transmedia en la concepción y el desarrollo de historias con una finalidad periodística constituye un ejercicio adecuado para conocer estas dinámicas de producción informativa y de adquisición de competencias.

\section{Método}

El artículo tiene el objetivo de conocer las características del periodismo transmedia y del transmedia literacy adquiridas por estudiantes de segundo año del Grado de Periodismo de la UAB en el desarrollo de la práctica de fin de curso de la asignatura "Escritura Periodística para Multimedia e Interactivos" (código de asignatura: 103109). El estudio explora los trabajos elaborados y analiza las competencias derivadas de la práctica educativa basada en la alfabetización transmedia.

Para evaluar las competencias transmedia y mediáticas, los alumnos han sido sometidos a una evaluación performativa, cuyo ejercicio consta en elaborar un reportaje transmedia sobre la Navidad en Barcelona con una duración máxima de 12 
horas (de 9 de la mañana a 9 de la noche). Por evaluación performativa, el estudio entiende una forma de regular, controlar y medir los resultados de un proceso educativo, como método para realizar críticas, comparaciones y exposiciones (Calderón y Oliveira-Junior, 2016, p. 43). La muestra, estudiada a través de un análisis de contenido, consta de una selección aleatoria de 28 trabajos elaborados por los alumnos entre 2013 y 2018. De cada año escolar (cuatro en total) fueron seleccionados siete trabajos que fueron elaborados en grupos de entre 4 y 6 estudiantes. El análisis de los ejercicios se ha complementado con tres grupos focales de cinco alumnos cada uno y llevados a cabo durante el mes de enero de 2019.

Grandío-Pérez (2016) hace una primera propuesta de indicadores para la evaluación de la transmedia literacy basada en las dimensiones de Ferrés \& Piscitelli (2012) y aplicada al contexto transmedia a partir de la propuesta de Jenkins (2010). La propuesta de indicadores de Grandío-Pérez ha sido utilizada para construir las preguntas semiestructuradas del guion de los grupos focales y para el trabajo de análisis de contenido.

\begin{tabular}{|l|l|}
\hline \multicolumn{1}{|c|}{ Dimensión } & \multicolumn{1}{c|}{ Indicadores } \\
\hline Lenguaje & $\begin{array}{l}\text { 1. Expresarse de manera multimodal. } \\
\text { 2. Establecer relaciones entre textos, códigos y medios. }\end{array}$ \\
\hline Tecnología & $\begin{array}{l}\text { 3. Desenvolverse en entornos hipermediales, transmedia y multimodales. } \\
\text { 4. Tener capacidad de manejo de herramientas multimediales y multimo- } \\
\text { dales. Recepción e interacción }\end{array}$ \\
$\begin{array}{l}\text { 5. Seleccionar la propia dieta mediática. } \\
\text { 6. Interaccionar con personas y colectivos en entornos cada vez más } \\
\text { plurales e interculturales. }\end{array}$ \\
\hline $\begin{array}{l}\text { 7. Trabajar de manera colaborativa en la elaboración de productos multi- } \\
\text { mediales y multimodales. } \\
\text { 8. Compartir y diseminar información a través de diferentes entornos } \\
\text { comunicativos. } \\
\text { 9. Tener una actitud responsable ante la identidad on-line/offline propia } \\
\text { y ajena. } \\
\text { 10. Contar con la capacidad de gestionar el concepto de autoría indivi- } \\
\text { dual y colectiva, y habilidad para aprovechar recursos como los creative } \\
\text { commons. }\end{array}$ \\
\hline
\end{tabular}


93 | Santiago Giraldo-Luque, Santiago Tejedor, Marta Portalés-Oliva y Ricardo Carniel-Bugs MONOGRÁFICO

\begin{tabular}{|c|c|}
\hline Dimensión & Indicadores \\
\hline Ideología & $\begin{array}{l}\text { 11. Evaluar la fiabilidad de las fuentes de información, y buscar, orga- } \\
\text { nizar, contrastar, priorizar y sintetizar informaciones procedentes de } \\
\text { distintos entornos. } \\
\text { 12. Tener una actitud ética a la hora de descargar productos en la red. } \\
\text { 13. Aprovechar las nuevas herramientas comunicativas para transmitir } \\
\text { valores y contribuir a la mejora del entorno. }\end{array}$ \\
\hline Estética & $\begin{array}{l}\text { 14. Relacionar las producciones mediáticas con otras manifestaciones } \\
\text { artísticas. } \\
\text { 15. Apropiarse y transformar producciones artísticas, potenciando la creati- } \\
\text { vidad, la innovación, la experimentación y la sensibilidad estética. }\end{array}$ \\
\hline
\end{tabular}

Tabla 2: Indicadores para la medición de la alfabetización transmedia.

Fuente: Grandio-Pérez (2016, p. 91).

Las variables relacionadas al transmedia storytelling aplicado al periodismo se han construido a partir de los trabajos de Alzamora y Tárcia (2014) y a la propuesta de evaluación de las NMLs realizada por Dawson y Siemens (2014), quienes valoran el transmedia a través del indicador "uso de tags, flujo de conversación a través de herramientas/espacios"3 (p. 297). En relación a las características del periodismo transmedia, el estudio incluye las dimensiones definidas por Jenkins (2009) y adaptadas al mundo periodístico por Martín y Rodríguez (2017, pp. 25-32). Las variables aplicadas al análisis de contenido son:

a. Expansión (Spreadability): La noticia debe poderse compartir a través de diversas redes on-line, además de que debe animar y permitir la interacción del lector.

b. Profundidad (Drillability): La noticia potencia que el lector profundice en la información a través de una invitación a la búsqueda de más información o a indagar en la noticia (Martín y Rodríguez, 2017). Scolari (2013b, p. 44) afirma que la participación del público será más profunda (drill) si existen extensiones y más contenidos de una misma noticia.

c. Continuidad (Continuity): El producto "mantiene el enfoque editorial y estilo periodístico al mismo tiempo que la historia se va expandiendo en diferentes medios y plataformas" (Martín y Rodríguez, 2017, p. 26). Esta característica 
se aplica a los trabajos de los alumnos cuando la información se encuentra en web y redes, y proporciona una experiencia unificada, continua y coherente (Jenkins, 2009).

d. Serialidad (Seriality): La historia se desarrolla en diferentes plataformas: “los fragmentos de información de la historia (...) se han dispersado no solo a través de múltiples segmentos dentro del mismo medio, sino a través de múltiples

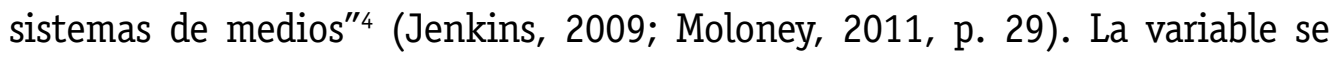
aplica si el contenido publicado en redes sociales se complementa con la información que es dada en otras plataformas, sin que se duplique la información.

e. Extractabilidad (Extractability): El contenido de la noticia puede extrapolarse a la vida diaria del lector (Moloney, 2011; (Scolari, 2013b, p. 40). Por ejemplo, en el contexto de la práctica de la asignatura, un vídeo tutorial de cómo hacer un decorado de navidad.

f. Creación de mundos (Worldbuilding): A través de las noticias se generan "universos" o "mundos" de las personas entrevistadas o de los lugares descritos (Scolari, 2013b, p. 40). "La historia principal puede desarrollarse, permitiendo historias alternativas basadas en diferentes personajes y circunstancias" ${ }^{5}$ (Moloney, 2011, p. 28).

g. Subjetividad (Subjectivity): La noticia se trata desde diferentes puntos de vista, representan "los puntos de vista múltiples de personajes o dimensiones dentro de la historia"6 (Moloney, 2011, p. 30). El análisis tomará en cuenta si la historia ofrece una variedad de puntos de vista sobre el tema elegido (el comprador y el vendedor, por ejemplo).

Se elimina de la propuesta original de Jenkins (2009) la variable de inmersión, ya que su complejidad excede el marco del trabajo. También se elimina la variable de multiplicidad (multiplicity) por encontrarse presente en todos los trabajos. Al mismo tiempo, se elimina la variable de realización (performance), ya que vincula la creación narrativa de fans con la historia original, siendo un elemento principal de la ficción y poco usual en el periodismo. Este conjunto de aspectos del transmedia propuestos por Jenkins se adaptaron a la investigación para funcionar como variables de análisis. La siguiente plantilla ${ }^{7}$ expone los ítems estudiados, tanto en los trabajos elaborados por los alumnos, como en los grupos focales. 
95 | Santiago Giraldo-Luque, Santiago Tejedor, Marta Portalés-Oliva y Ricardo Carniel-Bugs

\section{MONOGRÁFICO}

\begin{tabular}{|c|c|c|}
\hline Dimensión de análisis & Variable & Categorias \\
\hline \multirow{4}{*}{$\begin{array}{l}\text { Tratamiento informativo y } \\
\text { transmedia }\end{array}$} & Contiene imágenes & \multirow{4}{*}{$\begin{array}{l}\text { 0. No } \\
\text { 1. Sí }\end{array}$} \\
\hline & Contiene vídeo & \\
\hline & Contiene podcast & \\
\hline & Contiene herramientas interactivas & \\
\hline \multirow{10}{*}{$\begin{array}{l}\text { Alzamora y Tárcia, 2014; Daw- } \\
\text { son y Siemens, 2014) }\end{array}$} & ¿Qué herramientas interactivas? & Abierto \\
\hline & $\begin{array}{l}\text { Extensión a redes sociales } \\
\text { - Facebook } \\
\text { - Twitter } \\
\text { - YouTube } \\
\text { - Instagram }\end{array}$ & \multirow{4}{*}{$\begin{array}{l}0 . \text { No } \\
\text { 1. Sí }\end{array}$} \\
\hline & Interacción con audiencia & \\
\hline & Interacción entre redes sociales & \\
\hline & Uso de tags & \\
\hline & Texto con hipervínculos & $\begin{array}{l}\text { 0. No } \\
\text { 1. Sí, predomina con- } \\
\text { tenido propio } \\
\text { 2. Sí, predomina con- } \\
\text { tenido externo } \\
\text { 3. Ambas por igual }\end{array}$ \\
\hline & Tipo de Materiales & $\begin{array}{l}\text { 1. Propios principal- } \\
\text { mente } \\
\text { 2. Externos principal- } \\
\text { mente } \\
\text { 3. Ambos }\end{array}$ \\
\hline & Pies de foto y créditos & $\begin{array}{l}\text { 0. No } \\
\text { 1. Sí }\end{array}$ \\
\hline & Tratamiento fuentes & $\begin{array}{l}\text { 0. Documentales } \\
\text { 1. Personales } \\
\text { 2. Ambas }\end{array}$ \\
\hline & Evaluación cualitativa & $\begin{array}{l}\text { Comentarios en abier- } \\
\text { to por parte de los } \\
\text { investigadores }\end{array}$ \\
\hline
\end{tabular}


MONOGRÁFICO

\begin{tabular}{|c|c|c|}
\hline Dimensión de análisis & Variable & Categorías \\
\hline \multirow{3}{*}{ 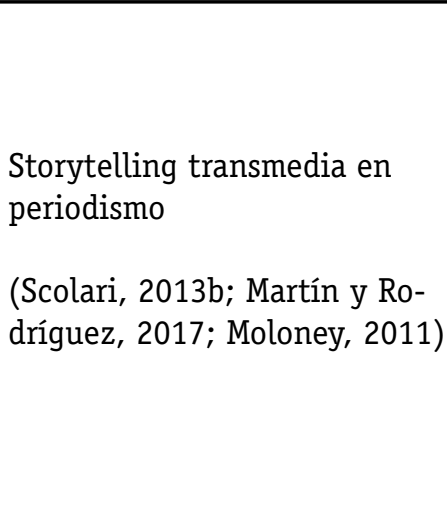 } & $\begin{array}{l}\text { 1. Expansión (Spreadability) } \\
\text { 2. Profundidad (Drillability) } \\
\text { 3. Multiplicidad (Multiplicity) } \\
\text { 4. Extractabilidad (Extractability) } \\
\text { 5. Creación de mundos (Worldbuilding) } \\
\text { 6. Subjetividad (Subjectivity) }\end{array}$ & $\begin{array}{l}\text { 0. No } \\
\text { 1. Sí }\end{array}$ \\
\hline & $\begin{array}{l}\text { Continuidad (Continuity) o } \\
\text { Serialidad (Seriality) }\end{array}$ & $\begin{array}{l}\text { 1. Continuidad } \\
\text { 2. Serialidad }\end{array}$ \\
\hline & Apreciación cualitativa & $\begin{array}{l}\text { Comentarios en abierto } \\
\text { por parte de los } \\
\text { investigadores }\end{array}$ \\
\hline
\end{tabular}

Tabla 3: La plantilla de análisis.

Fuente: Elaboración propia.

\section{Resultados}

Los resultados obtenidos, especialmente en los grupos focales, denotan que para el alumnado el concepto de historia adquiere importantes matices al pasar del escenario analógico al digital. Las reflexiones del alumnado inciden en la historia analógica como un relato más rígido, ordenado y lineal; frente a la versatilidad de los relatos en el ciberespacio.

\begin{tabular}{|l|l|}
\hline \multicolumn{1}{|c|}{ Definición de historia analógica } & \multicolumn{1}{c|}{ Definición de historia digital } \\
\hline "Es un relato que cuenta algo diferente". & "Lo más importante aparece al principio". \\
\hline $\begin{array}{l}\text { "Es algo ordenado, que posee planteamiento, } \\
\text { nudo y desenlace". }\end{array}$ & $\begin{array}{l}\text { "Puedes dirigir más hacia dónde va tu histo- } \\
\text { ria". }\end{array}$ \\
\hline "Explica las cosas en orden". & $\begin{array}{l}\text { "Es un tipo de historia que aprovecha el sopor- } \\
\text { te para introducir más opciones y recursos". }\end{array}$ \\
\hline $\begin{array}{l}\text { "Cuenta hechos relevantes } \\
\text { para el emisor y para el receptor". }\end{array}$ & $\begin{array}{l}\text { "Aporta dinamismo. No estás simplemente } \\
\text { leyendo un texto. El relato se mueve y tienes } \\
\text { forma de viajar en él". }\end{array}$ \\
\hline
\end{tabular}

Tabla 4: De la historia analógica a la historia digital.

Fuente: Elaboración propia a partir de los grupos focales realizados. 


\section{MONOGRÁFICO}

Al mismo tiempo, se detectó una confusión terminológica en la identificación de los elementos que definen a un relato transmedia. A nivel conceptual, los estudiantes no poseen un dominio pleno del concepto. El alumnado señala como rasgos diferenciadores del transmedia un conjunto de elementos y características que entremezclan aspectos estéticos, navegacionales, coyunturales, etc.

\begin{tabular}{|l|l|l|l|l|l|}
\hline Interactividad & Visualización & $\begin{array}{c}\text { Contraste y } \\
\text { verificación de } \\
\text { fuentes }\end{array}$ & Fiabilidad & Visual & $\begin{array}{c}\text { Único } \\
\text { (algo diferente, } \\
\text { distinto, } \\
\text { original) }\end{array}$ \\
\hline $\begin{array}{l}\text { Fragmentación } \\
\text { (no es algo } \\
\text { lineal) }\end{array}$ & Síntesis & Multiplataforma & Evolución & Novedad & $\begin{array}{l}\text { Crisis de } \\
\text { contenido }\end{array}$ \\
\hline Producción & $\begin{array}{l}\text { Diferentes } \\
\text { medios }\end{array}$ & $\begin{array}{l}\text { Diferentes } \\
\text { públicos }\end{array}$ & $\begin{array}{l}\text { Consumo } \\
\text { diferenciado }\end{array}$ & Posibilidades & Adaptación \\
\hline Audiovisual & Sencillo & $\begin{array}{l}\text { Cercano y fácil } \\
\text { para la gente }\end{array}$ & Diferente & Multiplataforma & $\begin{array}{l}\text { Tecnología } \\
\text { a favor del } \\
\text { contenido }\end{array}$ \\
\hline
\end{tabular}

Tabla 5: ¿Cuáles son los ingredientes de un relato transmedia?.

Fuente: Elaboración propia a partir de los grupos focales realizados.

Sobre la característica de la multimodalidad, el 100\% (28) de los reportajes transmedia analizados cuentan con imágenes y un $82 \%$ (23) contiene vídeos. Sin embargo, el audio es uno de los atributos con menor protagonismo. Tan solo un $25 \%$ (7) de los trabajos contienen recursos sonoros a pesar de que herramientas como Ivoox o SoundCloud, entre otras, ofrecen al alumnado oportunidades expresivas on-line. Por otro lado, el alumnado incorpora en sus trabajos una gran multitud de herramientas interactivas. En total, se han identificado 10 diferentes. La más utilizada es Infogram con un 29\% (16), seguida de Google Maps con 22\% (12) y Thinglink con $18 \%(10)$. Aun así, el $7 \%$ (2) de los trabajos no tenían ninguna herramienta multimedia, un 25\% (7) presentaba solo dos y un $33 \%$ (9) incluían en el contenido hasta tres herramientas multimedia. Únicamente, uno de los trabajos empleó cuatro herramientas multimediå ${ }^{8}$.

Estos datos de naturaleza cuantitativa pueden analizarse desde un enfoque cualitativo, ya que la inclusión de herramientas no equivale a una explotación correcta de las mismas. Por ejemplo, en la mayoría de los casos los mapas de Google sim- 
plemente mostraban los lugares desde donde se elaboraron los reportajes, pero los estudiantes no habían creado mapas interactivos con información adicional que ofreciera al usuario opciones de interacción. Asimismo, abundan los vídeos donde los alumnos tan solo graban el ambiente y no explotan capacidades expresivas del periodismo móvil (MoJo). Del mismo modo, no aprovechan los teléfonos móviles que tienen a su alcance para grabar y editar. La mayoría de las producciones audiovisuales incluidas en los reportajes son ambientes grabados que presentan un solo plano secuencia, sin edición, ni entrevistas, ni narrativa audiovisual. Sin embargo, existen algunas excepciones, como es el caso del trabajo Loterias y Navidad ${ }^{9}$ o Las Navidades del Cambio $^{10}$, cuyos reportajes audiovisuales incluyen una mayor concepción audiovisual, aunque el trabajo en su conjunto no es de los más destacados. Los estudiantes reconocieron en los grupos focales que existe una concentración en la producción de vídeos asociados al tipo de consumo propio, así como un uso básico de herramientas de visualización sin una intención real de profundizar en el contenido a partir de su implementación.

Sobre los materiales que los alumnos comparten, un 79\% (22) son contenidos de elaboración propia. Sin embargo, un 18\% (5) construyen sus contenidos a partir de materiales externos y de otras fuentes que obtienen en el ciberespacio. Finalmente, un $4 \%$ (1) combina ambos recursos (elaboración propia y recursos existentes en la Red) de forma equitativa.

Respecto al uso de hiperenlaces, aspecto crucial en la narrativa transmedia (Scolari, 2013b), un 32\% (9) no contienen hipervínculos. Además, cuando éstos existen, se usan para enlazar con contenido externo, en un 54\% (15) de los casos, frente a la promoción del material de propia plataforma, que se da solo en un $7 \%$ (2) de los casos. En un 7\% de los trabajos (2) se detecta un equilibrio entre ambos tipos de enlaces. El etiquetado de contenidos mediante palabras clave o etiquetas (tags) se emplea solo en un 14\% (4) de los ejercicios estudiados. En el grupo de discusión, los alumnos reconocieron la poca profundidad informativa en la gestión de la idea inicial del producto. Así, la articulación de historias entre diferentes plataformas no fue pensada como un elemento estratégico. Las redes sociales, por ejemplo, se incluyeron en algunos casos, "casi porque tenían que estar, pero no como una parte fundamental de la narración" (Estudiante 1). Respecto a la coordi- 


\section{MONOGRÁFICO}

nación entre la plataforma web y las redes sociales del mismo producto, en solo dos de los casos existe este tipo de interacción ${ }^{11}$ (Jenkins, 2009):

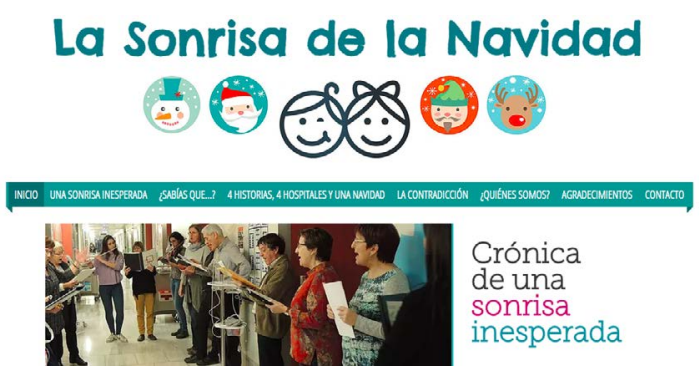

Tweets by @NavidadySonrisa

\section{Navidad y Sonrisa}

(0)NavidadySonrisa

María: "La mejor celebración de la Navidad seria que no hubiera niños en el Hospital". La entrevista completa aquí: alumnosuab.wixsite.com/lasonrisadelan...

Dec 23, 2016

Figura 1: Ejemplo de sección inmersiva "Tu camino" del trabajo "Navidad somos todos", elaborado por estudiantes.

A pesar de que la interacción y la conversación entre personas generada por la plataforma es uno de los principales elementos del producto transmedia (Alzamora y Tárcia, 2014; Dawson y Siemens, 2014), los estudiantes señalaron en el focus group que priorizan el contenido y el diseño (estético) del sitio web antes que la interacción. El estudio también permite señalar que solo el 21\% (6) de los trabajos fomenta la interacción con la audiencia. Por ejemplo, algunos ejercicios animan a la audiencia a que envíe sus propias fotos de navidad ${ }^{12}$, siguiendo la tendencia del crowdsourcing (Moloney, 2011; Carniel Bugs, 2014); otras permiten a la audiencia expresar su voz a través de encuestas ${ }^{13}$; fomentan los comentarios de lectores en Facebook $^{14}$, o la comprobación del número de la lotería de Navidad por parte de los internautas ${ }^{15}$. La presencia en redes sociales y plataformas sí es significativa, lideradas por YouTube (54\%), Twitter (39\%), Instagram (11\%) y Facebook (4\%).

Las propuestas analizadas no presentan, en su mayoría (75\%, 21 trabajos), características ligadas a la experimentación, la innovación y la creación artística en la concepción y el desarrollo del producto (Grandío-Pérez, 2016). Sin embargo, siete de los trabajos estudiados (25\%) aportan algún tipo de innovación. Dentro de ellos destacan los casos: Repartiendo ilusión ¿Cómo viven los niños desfavorecidos la navidad?16, que realiza una cobertura on-line durante la jornada en diferentes 
formatos multimodales, Navidad Low Cost $^{17}$ y Barcelona Interactiva Navidad 2.0 -Tradiciones i tecnología, que a partir de un tema transversal vinculan narraciones en diferentes plataformas. También es representativo el trabajo Navidad somos $\operatorname{todos}^{18}$, que invita al lector a interactuar para seguir la historia periodística, acercándose al formato inmersivo.

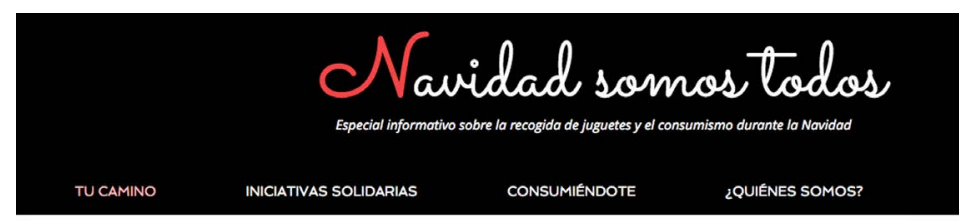

¿QUÉ CAMINO ESCOGES TÚ?

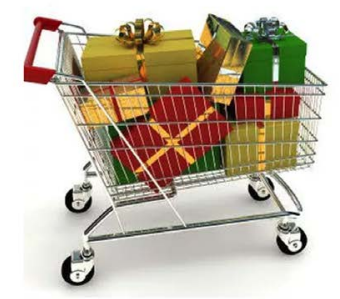

CONSUME

Elige ser uno más

Figura 2: Captura de la portada del trabajo "La Sonrisa de la Navidad", elaborada por estudiantes.

Cabe señalar, sin embargo, que los estudiantes reconocieron en los grupos focales que la práctica les había permitido desarrollar el sentido estético de la alfabetización transmedia y dialogar en grupo sobre su representación. Si bien los productos no implican altos contenidos de innovación en su gestación, sí existió un trabajo importante en la concepción del diseño del sitio web creado, así como en la valoración creativa del propio grupo.

Con relación al tratamiento de las fuentes de información (Alzamora y Tárcia, 2014; Grandío-Pérez, 2016), se detecta un hábito negativo de no precisar la procedencia de la información, ni citar explícitamente las fuentes de la misma. Los trabajos solo cuentan con pies de foto en los materiales gráficos de un 64\% (18) de los productos, un porcentaje bajo para la importancia de una correcta atribución del copyright en entornos digitales y en el periodismo (Grandío-Pérez, 2016). Con- 


\section{MONOGRÁFICO}

cretamente, un 9\% (5) no alude nunca a la procedencia de información, un 18\% (10) la menciona puntualmente y un $24 \%$ (13) siempre lo hacen. Para la elaboración de sus trabajos, el alumnado consulta en un 61\% (17) fuentes personales, y tan solo un $11 \%$ (3) incorpora fuentes documentales. En un $29 \%$ (8) de los casos las fuentes son tanto personales como documentales. Por ello, la categoría de subjetividad perteneciente al periodismo transmedia (Moloney, 2011) también tiene un valor inferior al que podría haber obtenido si los alumnos hubieran explotado la inclusión de los diferentes puntos de vista sobre un tema en concreto.

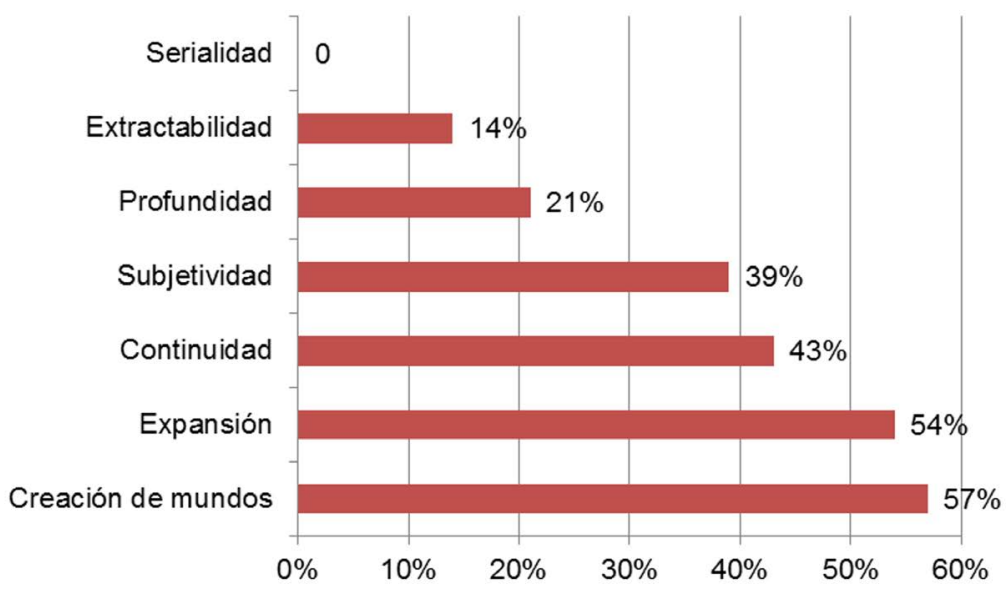

Figura 3: Presencia de categorías de transmedia storytelling

(Jenkins, 2009) en los trabajos analizados.

Fuente: Elaboración propia.

Como ejemplo de extractabilidad destacan dos trabajos. Por un lado, Gastronavidad en tiempos de crisis $^{19}$, que comparte una serie de recetas de platos navideños con los lectores. Por otro, la propuesta de divulgación científica de la probabilidad estadística de la Lotería en Navidad del trabajo Loterías y Navidad ${ }^{20}$. En ambos casos se trata de contenidos vinculados con la cotidianidad de los usuarios: "proporciona elementos para que los fans puedan llevarse aspectos de la historia a los espacios de su vida cotidiana"21 (Moloney, 2011, p. 28). La discusión sobre esta variable en los grupos focales permitió a los estudiantes reconocer una función social en su práctica periodística: “Algunos de los productos que realizamos en la práctica 
sí pueden extrapolarse a la vida cotidiana de la ciudadanía. Creo que pueden tener una capacidad de influencia sobre el público" (Estudiante 4). Al mismo tiempo, reconocían un impacto periodístico o de entretenimiento, pero no concebían una capacidad del producto de generar un proceso de incidencia social más allá de una posible generación de conciencia sobre un problema concreto asociado a la Navidad (desigualdad social, pobreza, consumismo, etc.).

De igual forma, los trabajos estudiados se inclinan por confeccionar el producto a partir de la variable de continuidad (Martín y Rodríguez, 2017), con un $43 \%$ (12) de uso, mientras que ninguno de los trabajos demuestra un uso del concepto de serialidad (Jenkins, 2009; Moloney, 2011). En cualquier caso, la mayoría de los ejercicios $57 \%$ (16) no utilizan un ecosistema propio de redes sociales que integren el producto transmedia. Sobre la variable de expansión es importante remarcar que un $46 \%$ (13) de los trabajos tenían iconos para permitir que fueran compartidos por redes, herramientas que concentraban toda la intención de continuidad en los trabajos analizados.

Por último, destacar el reconocimiento de las características ideológicas o valorativas en la producción del contenido informativo por parte de los estudiantes. Los estudiantes reconocen que existe un valor y un enfoque social asociado a la práctica y que, por tanto, el producto periodístico multimedia realizado en el ejercicio incide en la construcción de valores de la ciudadanía. Los estudiantes reconocieron, al mismo tiempo, el componente emotivo de la historia construida en un contexto temático específico (la Navidad), aunque algunos intentaban, justamente, "destruir el tópico y ofrecer otra manera de celebrar la navidad alejada del consumismo" (Estudiante 11).

\section{Discusión y conclusiones}

El estudio desarrollado pretendía analizar los parámetros considerados por los estudiantes de periodismo en la concepción y producción periodística de sus relatos transmedia. En este sentido, la investigación permite articular un conjunto de conclusiones que validan la hipótesis 01 ("El alumnado desatiende los aspectos relativos a la selección, contraste y verificación de los recursos multimedia que 


\section{MONOGRÁFICO}

utilizan en sus trabajos) y la 03 ("Los contenidos audiovisuales y de las redes sociales poseen un papel protagónico en la concepción de los proyectos informativos transmedia"). Sin embargo, la número 02 ("Los estudiantes atesoran una gran capacidad creativa en la ideación de propuestas narrativas con finalidad informativa que superan el modelo secuencial o lineal propio de la lógica del papel) queda únicamente parcialmente corroborada. Las principales conclusiones del estudio son las siguientes:

- Falta de autopercepción de competencias transmedia: No existe entre el alumnado una concepción clara de las características y el alcance del transmedia $\mathrm{y}$, por tanto, de sus posibilidades en la articulación de relatos con finalidades informativas. La posibilidad de construir mundos o universos con un talento comunicativo o informativo y que aproveche la profundidad de los recursos digitales es prácticamente inexistente. En los trabajos del alumnado falta la concepción y aplicación de aspectos vinculados con la interactividad, la hipertextualidad, el multimedia o la virtualidad.

- Predominio de los elementos visuales: Los recursos de naturaleza visual o audiovisual, ligados también al escenario creativo o estético de los estudiantes, sitúan a las imágenes y a los vídeos como los atributos más valorados y utilizados por el alumnado. Este aspecto, se corrobora al analizar las herramientas más usadas (Infogram, GoogleMaps y Thinglink) y a la preeminencia de YouTube como la red social con más presencia en los trabajos estudiados. Por lo tanto, la competencia audiovisual del alumnado es alta, sin embargo, el uso avanzado o crítico todavía no está presente en alumnos de segundo año de Periodismo.

- Propuestas de interacción reducidas: Solo en un $21 \%$ de los casos analizados se presentan propuestas que traten de interactuar con los usuarios. Este aspecto está estrechamente relacionado con el uso limitado de la narrativa hipertextual en el conjunto de trabajos.

- Apuesta por la producción de elaboración propia: Resulta de gran valor la amplia presencia (79\%) de contenidos de elaboración propia y de trabajo colaborativo entre los grupos que desarrollan la práctica propuesta (Martín y Rodríguez, 2017). Este aspecto de gran importancia en la formación de los futuros periodistas contrasta con la reducida combinación de herramientas y recursos 
on-line en un mismo trabajo. El aprendizaje colaborativo es clave en la creación de trabajos transmedia de alta complejidad y es una metodología educativa esencial, que se ha visto validada por el carácter longitudinal de este estudio. Los investigadores consideran que es esencial el aprendizaje colaborativo en la formación superior y no solo en niveles educativos inferiores.

- Protagonismo de las redes sociales: Las redes sociales destacan en el conjunto de las propuestas desarrolladas. Entre ellas, YouTube ocupa un papel estelar, aspecto que consolida la importancia conferida al audiovisual. No obstante, la inclusión de este tipo de plataformas, pese a ser amplia, advierte de una concepción de las mismas como espacios autónomos que no se coordinan o vehiculan con la plataforma web principal (ni directa ni indirectamente).

- Escasa apuesta por la innovación: La existencia de un número reducido de trabajos que destaquen por su componente innovador (únicamente un $25 \%$ del total) denota la necesidad de promover la experimentación con nuevos formatos a partir de las plataformas existentes y de la lógica del transmedia. Por lo tanto, la creatividad también es una de las competencias que necesitan ser promovidas en la carrera de Periodismo.

- Reconocimiento del contexto de transmisión de valores a partir de una historia periodística: Los estudiantes reconocen en sus trabajos un contenido ideológico a través del cual se transmiten valores sociales a los usuarios de la plataforma creada. A pesar del escaso alcance real de los ejercicios realizados, la identificación del rol social del periodista conecta con la construcción de un pensamiento crítico audiovisual asociado al transmedia literacy.

- Competencia instrumental versus poca criticidad: El conjunto de resultados obtenidos permite concluir que el alumnado posee un dominio técnico muy avanzado de las herramientas. Sin embargo, esta capacidad técnica no garantiza un uso periodístico por un lado, y crítico, por otro, de las mismas. Se plantea, por tanto, la necesidad de reformular las estrategias pedagógicas (Martínez y Echeverría, 2009) y, por ende, el diseño curricular de los planes de estudio para conferir un mayor protagonismo a las competencias transmedia de forma transversal y desde diferentes enfoques, con el objetivo de fomentar el pensamiento crítico de los estudiantes de Periodismo para que puedan afrontar los retos mediáticos actuales. 
105 | Santiago Giraldo-Luque, Santiago Tejedor, Marta Portalés-Oliva y Ricardo Carniel-Bugs

MONOGRÁFICO

\section{Referencias}

Alzamora, G. y Tárcia, L. (2014). Proposed methodology for transmedia news stories analysis: a comparative study of The Float Project (2009/10) in Brazil and The Great British Property Scandal: Every Empty Counts (2012). En H. Moura, R. Sternberg, R. Cunha, C. Queiroz, Z. Zeilinger (Eds.). Proceedings of the Interactive Narratives, New Media \& Social Engagement International Conference. Recuperado de http://interactiveconference.spanport.utoronto. $\mathrm{ca} /$ resources/Alzamora.pdf

AIMC - Asociación para la Investigación de Medios de Comunicación (2017): $19^{\circ}$ Navegantes en la Red. Madrid - España: AIMC. Recuperado de https://www. aimc.es/a1mc-c0nt3nt/uploads/2017/05/macro2016.pdf

Carrera Álvarez, P., Limón Serrano, N., Herrero Curiel, E., \& Sainz de Baranda Andújar, C. (2014). Transmedialidad y ecosistema digital. Historia y Comunicación Social, 18, 535-545. https://doi.org/10.5209/rev HICS.2013.v18.44257

Calderón, A.I.; De Oliveira-Junior, R.G. (2016). Evaluación por resultados y la cultura de la "performatividad": la evaluación docente en la escuela pública del estado de San Pablo (Brasil). Revista Iberoamericana de Evaluación Educativa, 7, (2e), 43-52. https://revistas.uam.es/index.php/riee/article/view/3112

Carniel Bugs, R. (2014). El 'crowdsourcing': La unión puede hacer la fuerza. En S. Tejedor y J.M. Pérez Tornero (Dirs.). Escribir para la Red. Reflexiones sobre la nueva (y vieja) escritura informativa 'online' (pp. 290-296). Bellaterra: Gabinete de Comunicación y Educación. Recuperado de http://www. gabinetecomunicacionyeducacion.com/sites/default/files/field/adjuntos/ libro escribir para la red $0 . p d f$

Cascajosa Virino, C. y Molina Cañabate, J. P. (2017). Narrativas expandidas entre la tradición y la innovación: construyendo el universo transmedial de El Ministerio del Tiempo. Tropelías. Revista de Teoría de la Literatura y Literatura Comparada, 27, 120-135. D0I: https://doi.org/10.26754/ojs tropelias/ tropelias.2017271544

Castells, M. (2003). La galàxia Internet: Reflexiones sobre internet, empresa y sociedad. Barcelona - España: Random House.

Comisión Europea (2016). Special Eurobarometer 438: E-Communications and the DigitalSingle Market. Bruselas - Bélgica: Comisión Europea. Recuperado de http:// 
ec.europa.eu/information society/newsroom/image/document/2016-22/ sp438 eb84 2 ecomm summary en 15829.pdf

Costa Sánchez, C. (2013). Narrativas Transmedia Nativas: Ventajas, elementos de la planificación de un proyecto audiovisual transmedia y estudio de caso. Historia y Comunicación Social, 18, 561-574. D0I: http://dx.doi.org/10.5209/ rev HICS.2013.v18.44349

Dawson, S. y Siemens, G. (2014). Analytics to literacies: The development of a learning analytics framework for multiliteracies assessment. The International Review of Research in Open and Distributed Learning, 15(4), 284-385. D0I: http://dx.doi.org/10.19173/irrodl.v15i4.1878

Francese, P. (2003). Trend Ticker: Ahead of the Next Wave. AdAge. Recuperado de: https://adage.com/article/american-demographics/trend-ticker-aheadwave $/ 44208$

Ferrés, J. y Piscitelli, A. (2012). La competencia mediática: propuesta articulada de dimensiones e indicadores. Comunicar, 38(19), 75-82. DOI: https://doi. org/10.3916/C38-2012-02-08

García Carrizo, J. y Heredero Díaz, 0. (2015). Propuesta de un modelo genérico de análisis de la estructura de las narrativas transmedia. Revista ICON014, 13(2), 260-285. D0I: https://doi.org/10.7195/ri14.v13i2.745

Gardner, H. y Davis, K. (2014). Generación App: cómo los jóvenes gestionan su identidad, su privacidad y su imaginación en el mundo digital. Barcelona: Grupo Planeta.

Grail Research (2011). Consumers of Tomorrow. Insights and Obervations About Generation Z. Recuperado de http://www.integreon.com/pdf/Blog/Consumers of Tomorrow Insights and Observations About Generation Z 246.pdf

Grandío-Pérez, M. M. (2016). El transmedia en la enseñanza universitaria. Análisis de las asignaturas de educación mediática en España (2012-2013). PalabraClave, 19(1), 85-104. D0I: http://dx.doi.org/10.5294/pacla.2016.19.1.4

Jenkins, H. (2003). Transmedia Storytelling. MIT Technology Review. Recuperado de https://www.technologyreview.com/s/401760/transmedia-storytelling

Jenkins, H. (2006). Convergence culture: When old and new media collide. Nueva York - EEUU: NYU Press.

Jenkins, H. (2008). Convergence Culture. La cultura de la convergencia de los medios de comunicación. Barcelona - España: Paidós. 
107 | Santiago Giraldo-Luque, Santiago Tejedor, Marta Portalés-Oliva y Ricardo Carniel-Bugs MONOGRÁFICO

Jenkins, H. (2009). The Revenge of the Origami Unicorn: Seven Principles of Transmedia Storytelling. Recuperado de http://henryjenkins.org/blog/2009/12/revenge of the origami unicorn.html

Jenkins, H. (2010). Transmedia Education: the 7 Principles Revisited. Recuperado de http://henryjenkins.org/blog/2010/06/transmedia education the 7 pri. $\underline{\text { html }}$

Kinder, M. (1991). Playing with Power in Movies, Television, and Video Games: From Muppet Babies to Teenage Mutant Ninja Turtles. Berkeley y Los Ángeles - EEUU: University of California Press.

Klastrup, L. y Tosca, S. (2004). Transmedial worlds-rethinking cyberworld design. 2004 International Conference on Cyberworlds (pp. 409-416). Recuperado de https://ieeexplore.ieee.org/document/1366205/

Larrondo Ureta, A. (2016). El relato transmedia y su significación en el periodismo. Una aproximación conceptual y práctica. Trípodos, (38), 31-47.

Leino, K., Linnakyla, P. y Malin, A. (2004). Finnish students' multiliteracy profiles. Scandinavian Journal of Educational Research, 48(3), 251-270. D0I: https:// doi.org/10.1080/00313830410001695727

Lévy, P. (1999). ¿Qué es lo virtual?. Barcelona - España: Paidós Ibérica.

Lévy, P. (2007). Cibercultura: la cultura de la sociedad digital. Barcelona - España: Anthropos.

Marfil-Carmona, R. (2013). Interactividad digital y estrategias narrativas en la publicidad audiovisual de Manos Unidas y Unicef. Historia y Comunicación Social, 18, 169-181. D0I: http://dx.doi.org/10.5209/rev HICS.2013.v18.43957 Martín, M. y Rodríguez, S. (2017). Manual de Periodismo Transmedia: Introducción y orientación al desarrollo de reportajes periodísticos transmedia. Trabajo de grado, Universidad Nacional de Córdoba. México.

Martínez Clares, P., \& Echeverría Samanes, B. (2009). Formación basada en competencias. RIE. Revista de Investigación Educativa, vol. 27, núm. 1, 125147. Recuperado de: https://revistas.um.es/rie/article/view/94331/102961

McCrindle, M. y Wolfinger, E. (2010). The ABC of XYZ: Understanding the Global Generations. Sydney: UNSW Press.

Moloney, K. T. (2011). Porting transmedia storytelling to journalism. Trabajo de fin de Máster, University of Denver. 
Palsa, L. y Ruokamo, H. (2017). Behind the concepts of multiliteracies and media literacy in the renewed Finnish core curriculum: A systematic literature review of peer-reviewed research. Seminar.net, 11(2), 101-119. Recuperado de https://journals.hioa.no/index.php/seminar/article/view/2354/2184

Pérez Latorre, 0. (2015). Alfabetización videolúdica y aprendizaje informal. Apuntes para una investigación. Working Paper Transmedia Literacy H2020. Recuperado de http://www.academia.edu/16573965/Alfabetizaci\%C3\%B3n videol\%C3\%BAdica y aprendizaje informal. Apuntes para una investigaci\%C3\%B3n

Pérez Tornero, J.M. y Celot, P. (2009). Study on current trends and approaches to media literacy in Europe. Bruselas - Bélgica: Comisión Europea. Recuperado de http://ec.europa.eu/assets/eac/culture/library/studies/literacy-trendsreport en.pdf

Piscitelli, A. (2002). Ciberculturas 2.0 en la era de las máquinas inteligentes. Barcelona - España: Paidós Ibérica.

Piscitelli, A., Adaime, I. y Binder, I. (Comp.) (2010). El Proyecto Facebook y la posuniversidad. Sistemas operativos sociales y entornos abiertos de aprendizaje. Madrid: Fundación Telefónica; Barcelona: Ariel. Recuperado de http://www. codajic.org/sites/www.codajic.org/files/El\%20Proyecto\%20Facebook.pdf

Postman, N. (1970). The Reformed English Curriculum. En A.C. Eurich (Ed.) High School 1980: The Shape of the Future in American Secondary Education (pp. 160-168). New York: Pitman.

Pratten, R. (2011). Getting Started in Transmedia StoryTelling: A Practical Guide for Beginners. Londres - Reino Unido: CreateSpace.

Scolari, C. A., \& Establés, M. J. (2017). El ministerio transmedia: expansiones narrativas y culturas participativas. Palabra clave, 20(4), 1008-1041. D0I: 10.5294/pacla.2017.20.4.7

Scolari, C. A. (2013a). Media Evolution. Emergence, Dominance, Survival and Extinction in the Media Ecology. International Journal of Communication, 7, 1418-1441. Recuperado de http://ijoc.org/index.php/ijoc/article/view/1919 Scolari, C. A. (2013b). Narrativas transmedia: cuando todos los medios cuentan. Barcelona - España: Grupo Planeta.

Scolari, C. A. (2016). Alfabetismo transmedia: Estrategias de aprendizaje informal y competencias mediáticas en la nueva ecología de la comunicación. Telos: 
109 | Santiago Giraldo-Luque, Santiago Tejedor, Marta Portalés-Oliva y Ricardo Carniel-Bugs

MONOGRÁFICO

Revista de pensamiento sobre Comunicación, Tecnología y Sociedad (293), 13-23. Recuperado de https://repositori.upf.edu/bitstream/handle/10230/27788/ Scolari Telos alfa.pdf? sequence $=1$

Tejedor, S. (2007). Periodismo "mashup". Combinación de recursos de la web social con una finalidad ciberperiodística. Anàlisi: quaderns de comunicació $i$ cultura, (35), 17-26. Recuperado de http://www.raco.cat/index.php/Analisi/article/ view/74252

Toffler, A. (1980). The Third Wave. Nueva York - EEUU: Bantam Books.

Tramullas, J. (2016). Hannibal ad portas, o los futuros perfiles profesionales de la información. El profesional de la información, 25(2), 157-162. D0I: http:// dx.doi.org/10.3145/epi.2016.mar.01

UNESCO (2013). Global Media and Information Literacy Assessment Framework: Country Readiness and Competencies. Paris: Unesco.

Vázquez García, Juan A. (2015). Nuevos escenarios y tendencias universitarias. Revista de Investigación Educativa, 33(1), 13-26.D0I: http://dx.doi. org/10.6018/rie.33.1.211501

Wolton, D. (2000). Internet, ¿y después? Una teoría crítica de los nuevos medios de comunicación. Barcelona - España: Gedisa.

\section{Notas}

[1] Programa de investigación e innovación de la UE Horizon 2020 (Contrato n. 645238). Consultar: https://transmedialiteracy.org

[2] Proyecto "New Media Literacies. Learning in a participatory culture", de la USC Annenberg School of Communication and Journalism. Consultar: http://www.newmedialiteracies.org

[3] Traducción propia del inglés: "Use of tags, conversation flow across tools/spaces".

[4] Traducción propia: "the chunks of meaningful and engaging story information have been dispersed not simply across multiple segments within the same medium, but rather across multiple media systems".

[5] Traducción propia: "The main story can unfold, allowing alternate stories based on different characters and circumstances".

[6] Traducción propia: "The views of multiple characters or dimensions within the story".

[7] Consultar datos y plantilla de análisis: http://bit.ly/2nZB6WF

[8] Consultar: http://loteriesinadal.esy.es/

[9] Consultar: http://loteriesinadal.esy.es/que-faria-amb-el-gordo/

[10] Consultar: http://navidadesdelcambio.blogspot.com.es/p/pagina-2.html 
Competencias transmedia en estudiantes de Periodismo... | 110

MONOGRÁFICO

[11] Consultar: http://alumnosuab.wixsite.com/lasonrisadelanavidad

[12] Consultar: http://elnadalalesescoles.blogspot.com.es/

[13] Consultar: http://nadallowcost.blogspot.com.es/

[14] Consultar: http://tradicionsbcn.wixsite.com/nadalinteractiu

[15] Consultar: http://loteriesinadal.esy.es/

[16] Consultar: https://repartiendoilusion.wordpress.com/un-juguete-una-sonrisa/

[17] Consultar: http://nadallowcost.blogspot.com.es/

[18] Consultar: http://dejandohuella365.wixsite.com/navidadsomostodos

[19] Consultar: http://gastronadal.blogspot.com.es/p/receptes.html

[20] Consultar: http://loteriesinadal.esy.es/probabilitat/

[21] Traducción propia: "provides something for that fan to then take aspects of that story with him or her into the spaces of his or her everyday life".

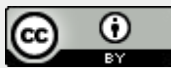

Este obra está bajo una licencia de Creative Commons Reconocimiento 4.0 Internacional. 